\title{
Thin Film Manganite-Metal Interconnection and "Loop Effect" Studies in CMR-Based High Magnetic Field Sensors
}

\author{
S. BAleviČiuss ${ }^{a, b, *}, \mathrm{~V}$. StankeviČ ${ }^{a, b}$, N. ŽUrauskiené ${ }^{a, b}$, Č. ŠImkeviČIUS ${ }^{a, b}$, \\ O. Liebfried ${ }^{a, c}$, M. LÖFfler ${ }^{d}$, M. Schneider ${ }^{c}, A$. Abrutis ${ }^{e}$ And V. PlaušinaitienĖ $\dot{A}^{a, e}$ \\ ${ }^{a}$ Semiconductor Physics Institute, A. Goštauto 11, Vilnius, Lithuania \\ ${ }^{b}$ Vilnius Gediminas Technical University, Sauletekio 11, Vilnius, Lithuania \\ ${ }^{c}$ French-German Research Institute of Saint Louis (ISL) \\ 5, rue Général Cassagnou, F-68301 Saint-Louis, France \\ ${ }^{d}$ Gelsenkirchen University of Applied Sciences \\ Neidenburger Str. 10, D-45877 Gelsenkirchen, Germany \\ ${ }^{e}$ Vilnius University, Naugarduko 24, LT-03225 Vilnius, Lithuania
}

\begin{abstract}
The design, technology and main characteristics of Ag contacts as well as "loop effect" peculiarities of colossal magnetoresistance $B$-scalar high magnetic field sensor based on $\mathrm{La}_{1-x}(\mathrm{Ca}) \mathrm{Sr}_{x} \mathrm{MnO}_{3}$ films used for measuring high magnetic field pulses are presented.
\end{abstract}

PACS numbers: 75.47.Gk, 75.47.Lx, 85.70.Ay

\section{Introduction}

It was demonstrated that the colossal magnetoresistance (CMR)- $B$-scalar sensor can be successfully used for high pulsed magnetic field measurements $[1,2]$. The sensor is a two-terminal device with thin manganite film as material sensitive to magnetic flux density $(B)$ magnitude. For such devices it is necessary that interconnection between electrode and thin film would be of low resistance with linear voltage-current characteristic. Moreover, the "loop effect" (LE) induced in circuit sensor-two current leads has to be much smaller than response to magnetic field. The "loop effect" depends on the time derivative of the magnetic field, and therefore, it sets an upper limit to the operational frequency of the device.

In this paper the properties of interconnection between thin $\mathrm{Ag}$ and $\mathrm{La}_{1-x}(\mathrm{Ca}) \mathrm{Sr}_{x} \mathrm{MnO}_{3}$ films and investigations concerning LE in CMR-B-scalar sensors are presented.

\section{Experimental results and discussion}

2.1. Interconnection $\mathrm{Ag}-\mathrm{La}_{1-x}(\mathrm{Ca}) \mathrm{Sr}_{x} \mathrm{MnO}_{3}$

The thin Ag film contact areas were fabricated by thermal deposition of $\mathrm{Ag}$ charge placed in $\mathrm{W}$ melting-pot at

\footnotetext{
* corresponding author; e-mail: sbal@pfi.lt
}

$\approx 10^{-5} \mathrm{kPa}$ vacuum. During $\mathrm{Ag}$ deposition the temperature of manganite film prepared by metalorganic chemical vapor deposition (MOCVD) technique on "Lucalox" substrate was $150-200^{\circ} \mathrm{C}$. The thickness of $\mathrm{Ag}$ film was changed from 0.2 to $1 \mu \mathrm{m}$. After Ag deposition the samples were heated during $20 \mathrm{~min}$ in oxygen atmosphere at $420-450^{\circ} \mathrm{C}$ temperature. Ag-electrode areas have square shape $(1 \times 1 \mathrm{~mm})$ and are separated in substrate plane at distance varying from $50 \mu \mathrm{m}$ to $3 \mathrm{~mm}$.

Investigation of contact resistance $\left(R_{\mathrm{c}}\right)$ vs. temperature $(T)$ dependence in the range $25 \div 300 \mathrm{~K}$ was performed using "four-probe" method. Typical results of this investigation obtained on polycrystalline $\mathrm{La}-\mathrm{Ca}-\mathrm{MnO}_{3}$ film are presented in Fig. 1.

Figure 1a shows that resistance $R=f(T)$ curves corresponding to manganite film (1) and $\mathrm{Ag}$ contact (2) demonstrate typical ferromagnetic to paramagnetic phase transition behaviour, however, the absolute value of contact resistance $\left(R_{\mathrm{c}}\right)$ is about $10^{4}$ times smaller than total film resistance $\left(R_{\mathrm{f}}\right)$. Moreover, critical temperature $\left(T_{\mathrm{m}}\right)$ at which resistance has its highest value is about $50 \mathrm{~K}$ higher in case of $R_{\mathrm{c}}$ vs. $T$ dependence. In order to clear up the nature of this phenomenon, simultaneously with deposition of contact areas, the surface of film was covered by ultrathin Ag film with thickness about $20 \mathrm{~nm}$. After thermal annealing at the same conditions as contact preparation the resistance of manganite film 

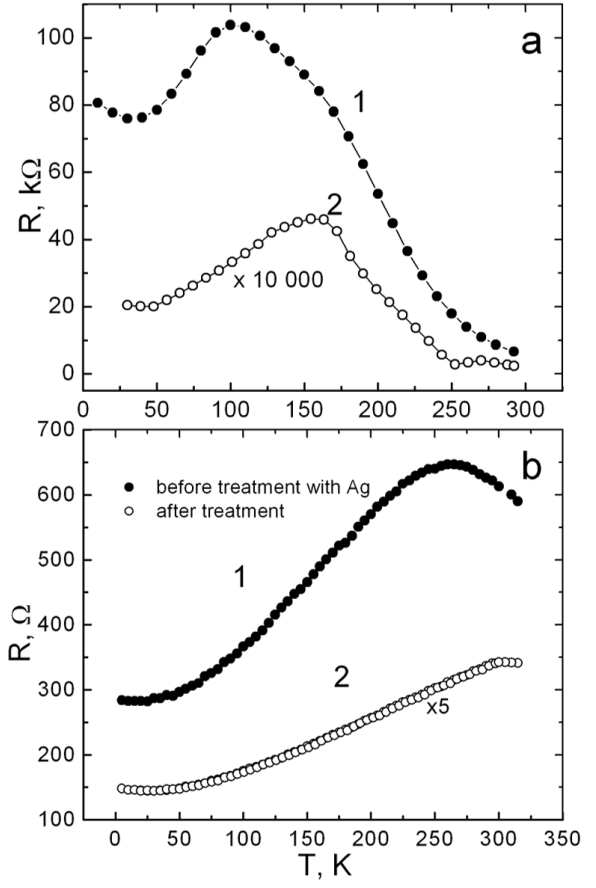

Fig. 1. Resistance vs. temperature dependence for $\mathrm{La}-\mathrm{Ca}-\mathrm{MnO}$ film (1) and $\mathrm{Ag}$ contact (2) (a) and for La-Sr-MnO film untreated (1) and treated by $\mathrm{Ag}(2)(\mathrm{b})$.

had decreased five times and $T_{\mathrm{m}}$ was shifted up to $50 \mathrm{~K}$ to higher temperatures (see Fig. 1b, curves 1 and 2).

The results presented in Fig. 1 show that Ag enables to create low resistance contact with $\mathrm{La}-\mathrm{Sr}-\mathrm{MnO}$ material. Thus it can be used for two-terminal resistive magnetic field sensor. From Fig. 1a it is evident that the interconnection between $\mathrm{Ag}$ and manganite film exhibits ferromagnetic to paramagnetic phase transition with lower resistance and higher $T_{\mathrm{m}}$ in comparison to the polycrystalline material. This can be explained assuming that $\mathrm{Ag}$ was introduced mainly at grain boundaries (GB) [3]. As a result, the net of GB are short-circuited at certain distance from the edge of $\mathrm{Ag}$ electrode. The region with short-circuited GBs has electric and magnetic properties similar to grain material with structure more perfect than GB. This assumption confirms experimental results obtained in [3] and presented in Fig. 1b. As can be seen, the significant decrease of resistance (10 times) and $T_{\mathrm{m}}$ shift $(\Delta T \approx 50 \mathrm{~K})$ to higher temperatures was obtained after introduction of small amount of $\mathrm{Ag}$ into $\mathrm{La}-\mathrm{Sr}-\mathrm{MnO}$ film.

\section{2. "Loop" effect}

The investigation of LE was performed using CMR$B$-scalar sensor having effective area $S=1 \times 0.05 \mathrm{~mm}^{2}$ and connected by $50 \mathrm{~cm}$ length twisted pair cable to the measurement circuit. The diameter of the wires and isolation thickness was $0.1 \mathrm{~mm}$ and $0.05 \mathrm{~mm}$, respectively. The length of each loop was $2.5 \mathrm{~mm}$. As magnetic field source the straight $10 \mathrm{~cm}$ length $\mathrm{Cu}$ lead carrying sinus waveform high current pulse was used. The sensor together with cable was placed near $\mathrm{Cu}$ lead at two positions: parallel and perpendicular to the lead. The LE was recorded as voltage transient $\left(V_{\mathrm{L} 0}\right)$ at the beginning of the current pulse (see Fig. 2a).

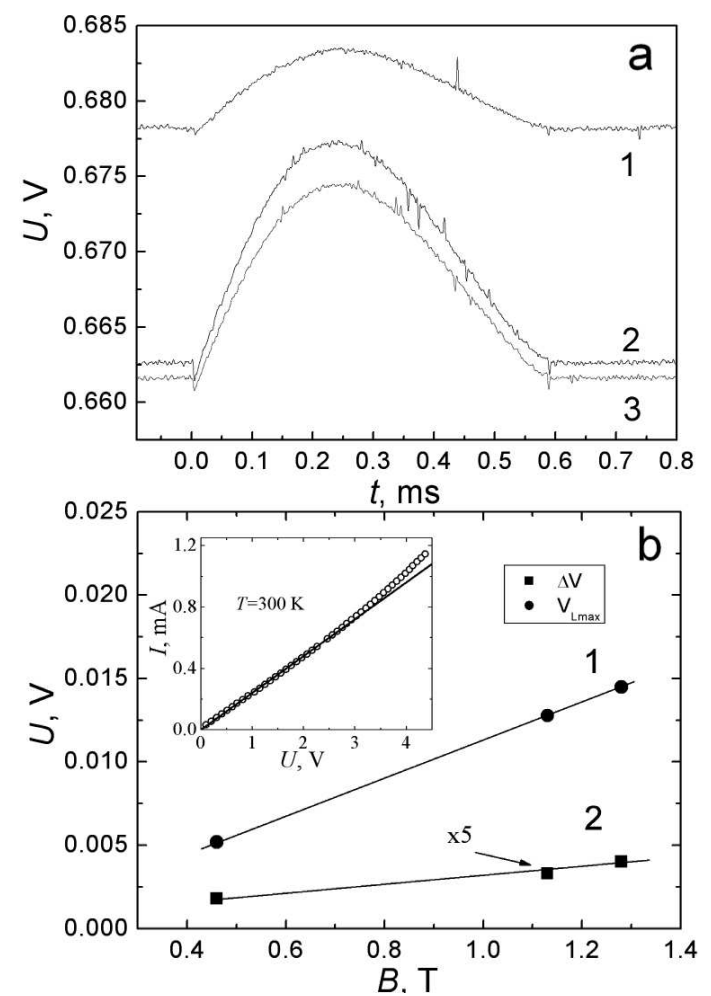

Fig. 2. Sensor's response to sinus waveform magnetic field pulses having different $B_{0}: 1-0.46 \mathrm{~T}, 2-1.13 \mathrm{~T}$, and $3-1.28 \mathrm{~T}(\mathrm{a})$. The $\Delta V$ and $V_{\mathrm{L} \max }(\times 5)$ as a function of $B_{0}$ and $\mathrm{DC}$ voltage-current characteristic (inset) of sensor at $300 \mathrm{~K}$ temperature (b).

The highest value of $V_{\mathrm{L} 0}$ was obtained when cable was in perpendicular position to the current lead due to non-homogeneous ( $B \sim 1 / x, x$ is coordinate) magnetic field along the twisted cable. In case when cable was placed in parallel to the current lead the $V_{\mathrm{L} 0}$ was approximately ten times less in comparison to the perpendicular position. For magnetic field pulse having half of sinus waveform ( $B=B_{0} \sin \omega t, \omega=\pi / T$, here $T$ is pulse duration) the voltage induced by "loop" effect $V_{\mathrm{L} 0}=B_{0} S_{\text {eff }} \omega \cos \omega t$ ( $S_{\text {eff }}$ is effective area of the "sensor-cable" system). The maximal value of this voltage $V_{\mathrm{L} 0 \max }=B_{0} S_{\text {eff }} \omega$ appeared at time instant $t=0$. At fixed $B_{0}$ and $S_{\text {eff }}$ amplitude $V_{\mathrm{L} 0 \max }$ depends on $\omega$. If the sensor is supplied by DC current, the accuracy of the measurement depends on the ratio beween voltage change $\Delta V$ due to CMR effect and $V_{\mathrm{L} 0 \max }$. For $\pm 5 \%$ accuracy the ratio $\Delta V / V_{\mathrm{L} 0 \max }=10$. Nominating $\Delta V / B_{0}=k_{1}$ and $V_{\mathrm{L} 0 \max } / B_{0}=k_{2}$, we obtained the following formula for calculation of maximal possible frequency $\left(f_{\max }\right)$, which can be measured using CMR- $B$-scalar sensor 


$$
f_{\max }=0.1\left(k_{1} / k_{2}\right) f_{m}
$$

where $f_{\mathrm{m}}$ is the fundamental frequency of the magnetic pulse, which was used for determination of coefficients $k_{1}$ and $k_{2}$. Figure $2 \mathrm{~b}$ presents $\Delta V$ and $V_{\mathrm{L} \max }$ as a function of $B_{0}$ obtained when voltage drop at the sensor was $0.8 \mathrm{~V}$ and $f_{\mathrm{m}}=0.833 \mathrm{kHz}$. The coefficients $k_{1}$ and $k_{2}$ calculated using data from Fig. $2 \mathrm{~b}$ was 11.34 and $0.625 \mathrm{mV} / \mathrm{T}$, respectively, that give $f_{\max } \approx 1.5 \mathrm{kHz}$ according to (1). The $f_{\max }$ can be increased biasing sensor by higher voltage, however this way has a limitation due to Joule's heating dissipated in magnetic material. In order to evaluate this heating we measured voltage-current characteristic (inset in Fig. 2b) of the sensor having dimensions $10^{3} \times 50 \times 0.4 \mu \mathrm{m}^{3}$ and obtained that up to $\approx 2.5 \mathrm{~V}$ the heating effect did not alter the resistance of the sensor. Thus using biasing by $2.5 \mathrm{~V}$ it is possible to increase $f_{\max }$ up to $5 \mathrm{kHz}$. Calculations using "loop" effect measurement data showed that effective area $S_{\text {eff }}$ is of the order of $0.12 \mathrm{~mm}^{2}$, while effective area of the sensor $S=0.05 \mathrm{~mm}^{2}$. This demonstrates that $S$ is about half of $S_{\text {eff }}$, consequently small dimensions of the CMR- $B$-scalar sensor enables to design low effective area sensor-cable system, which can operate at accuracy $\pm 5 \%$ or better up to $5 \mathrm{kHz}$ without cable shielding.

\section{Conclusions}

It was concluded that thin Ag films can be successfully used as electrodes for CMR- $B$-scalar sensor design. The $\mathrm{Ag}$ mainly penetrates into the grain boundaries regions of the polycrystalline thin manganite film. The "loop" effect and heating of the thin manganite film by bias current limits the upper operation frequency of unshielded sensor up to several $\mathrm{kHz}$.

\section{Acknowledgments}

The work was supported by the Lithuanian Science and Studies Foundation contact No. B-21/2008.

\section{References}

[1] S. Balevicius, N. Zurauskiene, V. Stankevic. S. Kersulis, J. Novickij, L.L. Altgilbers, F. Clarke, Acta Phys. Pol. A 107, 207 (2005).

[2] M. Schneider, R. Schneider, V. Stankevic, S. Balevicius, N. Zurauskiene, IEEE Trans. Magn. 43, 370 (2007).

[3] J. Li, Q. Huang, Z.W. Li, L.P. You, S.Y. Xu, C.K. Ong, J. Phys. Condens. Matter 13, 3419 (2001). 